\title{
New gene panel for aggressive prostate cancer
}

A panel of three genes in urine shows promise for determining which patients have an aggressive form of prostate cancer, according to a new paper.

Overtreatment is a major concern in prostate cancer as serum PSA screening cannot identify which tumours will become very aggressive and which are benign. The Progensa ${ }^{\circledR}$ (GEN-PROBE, CA, USA) PCA3 test is approved by the FDA for detecting prostate cancer in urine, but has limited use in distinguishing indolent from aggressive disease. Biomarkers to achieve this aim are urgently needed.

\section{4 ...serum PSA screening cannot identify which tumours will become very aggressive... 77}

Gisele Leyten and co-workers in the Netherlands examined gene expression in normal prostate and in different prostate cancer tissue samples (including low-grade, high-grade and castrationresistant prostate cancer) and identified 39 candidate biomarker genes that were upregulated in the disease. Leyten et al. tested these 39 candidate biomarkers using quantitative PCR and selected the 16 biomarkers that were most differentially expressed between cancer and noncancer patients and showed prostate-specific expression. Of these, eight genes-ONECUT2, HOXC4, HOXC6, DLX1, TDRD1, NKAIN1, MS4A8B, PPFIA2-were selected for clinical testing in 358 urinary sediments from men scheduled for initial or repeat prostate biopsies.

Among the 358 men whose urinary sediments were used for testing, 157 (44\%) were diagnosed with prostate cancer on biopsy and 93 (26\%) had prostate cancer with a Gleason score $\geq 7$. Leyten et al. used real-time quantitative PCR to examine expression of the eight potential biomarkers in the urinary sediments. They found that all of the potential biomarkers were able to distinguish prostate cancer from no prostate cancer and prostate cancer Gleason score $\geq 7$ from prostate cancer Gleason score $\leq 6$. Three of the biomarkers-HOXC6, DLX 1 and TDRD1 - had independent additional predictive value to serum PSA for the detection of Gleason score $\geq 7$. Bootstrap resampling confirmed that these three genes were useful as an adjunct to serum PSA for predicting biopsy Gleason score $\geq 7$ in urinary sediments.

The researchers found that their panel of genes was more accurate in predicting Gleason score $\geq 7$ in biopsies than was either the Progensa ${ }^{\circledR}$ PCA3 panel or serum PSA. They also found that combining

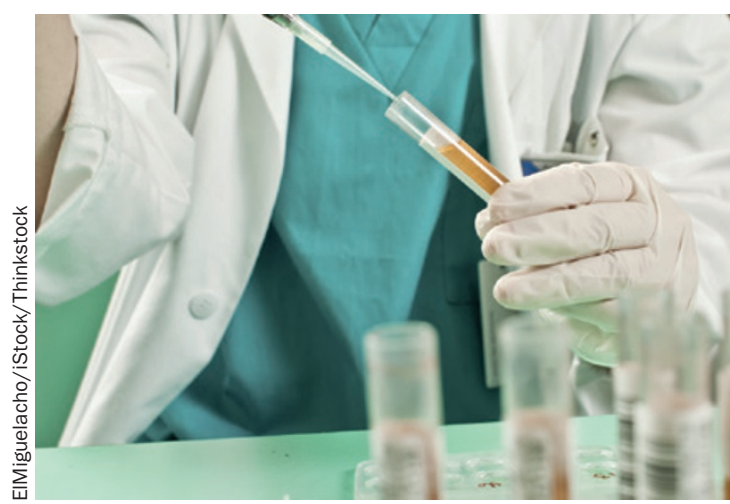

the three-gene panel with serum PSA improved its predictive accuracy further, and that the accuracy of the panel was maintained even in subgroups with low serum PSA concentrations.

The authors state that their urinary three-gene panel represents a promising tool for identifying patients with aggressive prostate cancer and that the combination of the urinary three-gene panel with serum PSA has great potential for the early diagnosis of patients with clinically significant prostate cancer.

Rebecca Kelsey

Original article Leyten, G. H. J. M. et al. Identification of a candidate gene panel for the early diagnosis of prostate cancer. Clin. Cancer Res. doi:10.1158/1078-0432.CCR14-3334 